\title{
Eribulin mesylate versus ixabepilone in patients with metastatic breast cancer: a randomized Phase II study comparing the incidence of peripheral neuropathy
}

\author{
Linda T. Vahdat • Agustin A. Garcia $\cdot$ Charles Vogel $\cdot$ Christine Pellegrino • \\ Deborah L. Lindquist • Nicholas Iannotti · Prashanth Gopalakrishna • \\ Joseph A. Sparano
}

Received: 4 April 2013/Accepted: 17 May 2013/Published online: 23 July 2013

(C) The Author(s) 2013. This article is published with open access at Springerlink.com

\begin{abstract}
Peripheral neuropathy is a common toxicity associated with tubulin-targeted chemotherapeutic agents. This Phase II study compares the incidence and severity of neuropathy associated with eribulin mesylate or ixabepilone in metastatic breast cancer (MBC). The primary objective was to assess the incidence of neuropathy; the study was designed to detect a difference in neuropathy rate of $35 \%$ for eribulin versus $63 \%$ for ixabepilone (odds ratio $0.316,80 \%$ power, 0.05 two-sided significance level). Eligibility criteria included: MBC; prior taxane therapy; at least one chemotherapy for advanced disease; no or minimal pre-existing neuropathy (Grade 0 or 1 ). The intent-to-treat population comprised 104 patients randomized $(1: 1)$ to eribulin mesylate $\left(1.4 \mathrm{mg} / \mathrm{m}^{2}, 2-5 \mathrm{~min}\right.$ intravenous on days 1 and 8$)$ or ixabepilone $\left(40 \mathrm{mg} / \mathrm{m}^{2}\right.$, $3 \mathrm{~h}$ intravenous on day 1) on a 21-day cycle. 101 patients
\end{abstract}

Previous presentations Data from this study were previously presented at the 34th Annual CTRC-AACR San Antonio Breast Cancer Symposium, San Antonio, TX, USA, December 6-10, 2011, and at the ASCO Breast Cancer Symposium, San Francisco, CA, USA, September 13-15, 2012.

\section{T. Vahdat ( $\square)$}

Weill Cornell Medical College, 425 East 61st Street, 8th Floor,

New York, NY 10065, USA

e-mail: 1tv2001@med.cornell.edu

\section{A. A. Garcia}

Keck School of Medicine of the University of Southern

California, Los Angeles, CA, USA

C. Vogel

Sylvester Comprehensive Cancer Center, University of Miami,

Miller School of Medicine, Deerfield Beach, FL, USA

C. Pellegrino $\cdot$ J. A. Sparano

Montefiore Medical Center, Albert Einstein College of

Medicine, New York, NY, USA in the safety population received a median of 5.0 eribulin and 3.5 ixabepilone cycles. Incidence of neuropathy (any grade) was 33.3 and $48.0 \%$, and peripheral neuropathy was 31.4 and $44.0 \%$ for eribulin and ixabepilone, respectively. After controlling for pre-existing neuropathy and number of prior chemotherapies, these differences were not significant. Compared with ixabepilone, fewer patients receiving eribulin discontinued treatment due to neuropathy (3.9 vs. $18.0 \%$ ) or adverse events (AEs) in general (11.8 vs. $32.0 \%)$. Time to onset of neuropathy was 35.9 weeks for eribulin and 11.6 weeks for ixabepilone, and time to resolution was 48 versus 10 weeks, respectively; other AEs were comparable. Objective responses were 15.4 versus $5.8 \%$ and clinical benefit rates were 26.9 versus $19.2 \%$. In conclusion, after controlling for preexisting neuropathy and number of prior chemotherapies, the differences in the incidence of neuropathy with eribulin and ixabepilone were not statistically significant. Onset of neuropathy tended to occur later with eribulin and resolve later.

\author{
D. L. Lindquist \\ US Oncology, Sedona, AZ, USA \\ N. Iannotti \\ Hematology-Oncology Associates of the Treasure Coast, Port \\ Saint Lucie, FL, USA \\ P. Gopalakrishna \\ Eisai Ltd, Hatfield, UK
}


Keywords Eribulin mesylate $\cdot$ Ixabepilone $\cdot$ Metastatic breast cancer $\cdot$ Peripheral neuropathy

$\begin{array}{ll}\text { Abbreviations } \\ \text { AE } & \text { Adverse event } \\ \text { CBR } & \text { Clinical benefit rate } \\ \text { CI } & \text { Confidence interval } \\ \text { CR } & \text { Complete response } \\ \text { CT } & \text { Computed tomography } \\ \text { CTCAE } & \text { Common Terminology Criteria for Adverse } \\ & \text { Events } \\ \text { DCR } & \text { Disease control rate } \\ \text { ECOG } & \text { Eastern Cooperative Oncology Group } \\ \text { EMBRACE } & \text { Eisai Metastatic Breast Cancer Study } \\ & \text { Assessing Physician's Choice Versus E7389 } \\ \text { FISH } & \text { Fluorescence in situ hybridization } \\ \text { HER2 } & \text { Human epidermal growth factor receptor 2 } \\ \text { HR } & \text { Hazard ratio } \\ \text { MBC } & \text { Metastatic breast cancer } \\ \text { MedDRA } & \text { Medical Dictionary for Regulatory Activities } \\ \text { ORR } & \text { Objective response rate } \\ \text { OS } & \text { Overall survival } \\ \text { PD } & \text { Progressive disease } \\ \text { PFS } & \text { Progression-free survival } \\ \text { PgR } & \text { Progesterone receptor } \\ \text { PN } & \text { Peripheral neuropathy } \\ \text { PNQ } & \text { Patient-reported neurotoxicity questionnaire } \\ \text { PR } & \text { Partial response } \\ \text { SD } & \text { Stable disease } \\ \text { TPC } & \text { Treatment of physician's choice } \\ \text { VPT } & \text { Vibration perception threshold } \\ & \end{array}$

\section{Introduction}

Eribulin mesylate $\left(\right.$ Halaven $^{\circledR}$, INNM: eribulin mesilate, E7389), a non-taxane microtubule dynamics inhibitor, is a structurally simplified, synthetic analog of the natural product Halichondrin B, isolated from the marine sponge Halichondria okadai $[1,2]$. By predominantly binding to high affinity sites on the growing plus (+) ends of microtubules, eribulin inhibits microtubule polymerization without affecting depolymerization, which in turn induces irreversible mitotic block at $\mathrm{G}_{2}-\mathrm{M}$ phase and apoptosis [1-6]. This mechanism of action is distinct from most other tubulintargeting chemotherapeutic agents currently in clinical use, including taxanes, vinorelbine, and epothilones [3, 5].

In the randomized Phase III Eisai Metastatic Breast Cancer Study Assessing Physician's Choice Versus E7389 (EMBRACE) trial, which involved patients with heavily pretreated locally recurrent or metastatic breast cancer (MBC), eribulin was compared with treatment of physician's choice
(TPC) [7]. Patients had received a median of four previous chemotherapy regimens including an anthracycline and a taxane. Patients who received eribulin mesylate $\left(1.4 \mathrm{mg} / \mathrm{m}^{2}\right.$, days 1 and 8 of a 21-day cycle) exhibited a significant improvement in median overall survival (OS) compared with TPC (13.1 vs. 10.6 months; hazard ratio [HR] 0.81; $95 \%$ confidence interval $[\mathrm{CI}] 0.66-0.99 ; p=0.041)$. An updated analysis of OS requested by regulatory authorities confirmed the significant increase in OS for eribulin compared with TPC (13.2 vs. 10.5 months; HR 0.81; $95 \%$ CI 0.67-0.96; nominal $p=0.014)$. This was the first Phase III monotherapy study to meet its primary endpoint of prolonged OS in this patient population. These results contributed in part to the Food and Drug Administration's approval of eribulin mesylate for treatment of patients with MBC who have previously received at least two chemotherapies for the treatment of metastatic disease and an anthracycline and a taxane in either the adjuvant or metastatic setting [8].

Peripheral neuropathy (PN) is a common toxicity associated with chemotherapy agents which target microtubules. It can also be a treatment-limiting factor for patients with heavily pre-treated MBC [9], and have a significant impact on patients' routine activities, functions, and behavior [10]. In EMBRACE, $35 \%$ of patients treated with eribulin experienced PN (all grades), with $8 \%$ and $<1 \%$ reporting Grade 3 and 4 PN, respectively [7]. For patients in the TPC arm, total incidence of PN was $16 \%$ (Grade 3, $2 \%$; no Grade 4 events) [7]. In two Phase II studies of eribulin in patients who had previously received an anthracycline and a taxane [11] or an anthracycline, a taxane, and capecitabine [12], incidences of PN were $26 \%$ (Grade 1/2) and 5-7 \% (Grade 3 ), with no Grade 4 events reported in either study.

Ixabepilone is an epothilone B analog, which induces microtubule stabilization [13]. It is indicated for treatment of locally advanced or MBC after failure of an anthracycline, a taxane, and capecitabine, or combined with capecitabine following anthracycline and taxane failure [14]. Ixabeplione has been investigated in three Phase II monotherapy studies [15-17] and included as a comparator in a Phase III study [18] involving patients with MBC. In two of the Phase II studies (ixabepilone in patients with $\mathrm{MBC}$ resistant to either a taxane [15] or an anthracycline, a taxane, and capecitabine [16]), 60-63\% of patients reported sensory PN (all grades); motor neuropathy was reported by $2-10 \%$ of patients and Grade $3 / 4$ events reported by $12-13 \%$ of patients $[15,16]$. In the third Phase II study, ixabepilone was administered as first-line therapy in taxane-naïve patients with MBC previously treated with an adjuvant anthracycline. Treatment with ixabepilone was associated with an incidence of sensory PN of $71 \%$ (all grades) and $20 \%$ (Grade 3; no Grade 4), and of motor neuropathy of $6 \%$ (5\% Grade 3; no Grade 4) [17]. In the Phase III study comparing paclitaxel, nanoparticle albumin-bound paclitaxel, and ixabepilone, with or without 
bevacizumab, as first-line therapy for $\mathrm{MBC}, \geq$ Grade 2 sensory $\mathrm{PN}$ was reported for $44 \%$ of patients treated with ixabepilone [18].

While these reports highlight that incidences of chemotherapy-induced PN differs between conventional agents, the precise underlying cellular mechanisms are unclear. In preclinical studies using mouse models of neuropathy, paclitaxel and ixabepilone at their equivalent maximum tolerated doses produced deficits in nerve conduction parameters and caused degenerative pathological changes, whereas eribulin did not affect conduction and caused milder, less frequent effects on cell morphology [19]. In in vitro studies, inhibition of microtubule-dependent axonal transport was reduced with eribulin compared with paclitaxel, ixabepilone, and vincristine [20]. These results suggest that eribulin may have lesser clinical impact upon nerve function and morphology than other tubulintargeting agents.

This Phase II, randomized study assessed the incidence and severity of neuropathy adverse events (AEs) in patients with locally recurrent or MBC after treatment with either eribulin or ixabepilone. Overall safety, tolerability, and efficacy profiles were also examined.

\section{Methods}

\section{Study design}

This Phase II, multicenter, randomized, open label study (Study E7389-G000-209; NCT00879086) recruited patients with locally recurrent or $\mathrm{MBC}$ who had received prior taxane therapy, at least one prior cytotoxic chemotherapy for advanced disease, and progressed during or after their last anti-cancer therapy. Patients were enrolled at 48 sites in the United States.

Patients were pre-stratified based on pre-existing neuropathy (National Cancer Institute Common Terminology Criteria for Adverse Events version 3.0 [CTCAE] Grade 0 or 1$)$ and number of prior chemotherapies $(\leq 3$ or $>3$ ). Patients were then randomized (1:1) to eribulin or ixabepilone treatment groups in a consecutive sequence.

All patients provided written informed consent. The study was conducted in accordance with the International Conference on Harmonization Guidelines for Good Clinical Practice, the Declaration of Helsinki, and the institutional review board/ethics committee at each site.

\section{Study objectives}

The primary objective was to assess the incidence of neuropathy AEs in patients treated with eribulin or ixabepilone, graded using the CTCAE. Neuropathy was based on a broad list of preferred terms for neuropathy and the following additional preferred terms: neuropathy, hyperesthesia, painful response to normal stimuli, pallanesthesia, and allodynia. As a sensitivity analysis, analysis of neuropathy was also performed based on a narrow definition of PN (combined term), including the following preferred terms: neuropathy peripheral, peripheral motor neuropathy, polyneuropathy, peripheral sensory neuropathy, peripheral sensorimotor neuropathy, demyelinating polyneuropathy, and paresthesia. The secondary safety objectives included: comparison of severity of neuropathy AEs (defined as for the primary objective), patient-reported neurotoxicity questionnaire (PNQ), and vibration sensitivity (vibration perception threshold [VPT]); time to onset and resolution of neuropathy; and overall safety and tolerability. Secondary efficacy objectives were objective response rate (ORR), clinical benefit rate (CBR), progression-free survival (PFS), and disease control rate (DCR).

\section{Patients}

Key inclusion criteria were: women at least 18 years old with histologically or cytologically confirmed carcinoma of the breast (locally recurrent or metastatic); prior taxane therapy and at least one prior cytotoxic chemotherapy for locally recurrent or $\mathrm{MBC}$; disease progression during or after last anti-cancer therapy; pre-existing neuropathy $<$ Grade 2; Eastern Cooperative Oncology Group performance status of 0-2; adequate renal, liver, and bone marrow function. Patients must have shown resolution of all prior chemotherapy or radiation-related toxicities to Grade $\leq 1$, except for alopecia, and the ability to complete the PNQ without assistance from study-site personnel.

Exclusion criteria included: prior ixabepilone or eribulin therapy; a history of diabetes mellitus concurrent diseases or conditions expected to interfere with neuropathy assessments; and significant cardiovascular impairment.

\section{Treatment}

Eribulin mesylate $1.4 \mathrm{mg} / \mathrm{m}^{2}$ (equivalent to eribulin $1.23 \mathrm{mg} / \mathrm{m}^{2}$ [expressed as free base]) was administered intravenously over 2-5 min on days 1 and 8 of each 21-day cycle, and ixabepilone (starting dose 32 or $40 \mathrm{mg} / \mathrm{m}^{2}$ as per approved labeling) as a $3-\mathrm{h}$ intravenous infusion on day 1 of each 21-day cycle. A reduced dose of ixabepilone at 32 or $20 \mathrm{mg} / \mathrm{m}^{2}$ was allowed for patients with mild or moderate hepatic impairment, respectively, according to approved labeling [14].

Treatment continued until unacceptable toxicity, progression of disease, or the investigator considered that discontinuation of therapy was in the patient's best interest. Dose delays and modifications were permitted to manage toxicities. 


\section{Concomitant medication}

Any medication considered necessary for the patient's welfare that was not expected to interfere with the evaluation of either study drug was permitted at the investigator's discretion. Palliative radiotherapy was permitted if the irradiated area involved $<10 \%$ of the bone marrow and was not to be used for tumor response assessment. Study treatment was delayed during palliative radiotherapy. Strong CYP3A4 inhibitors, other investigational drugs, and other anti-tumor therapies were prohibited during the study; mild or moderate CYP3A4 inhibitors, strong CYP3A4 inducers, and CYP3A4 substrates were administered with caution.

\section{Safety}

AEs were graded using CTCAE and coded according to the Medical Dictionary for Regulatory Activities. AEs reported here were considered treatment-emergent, i.e., they commenced on or after day 1 of cycle 1, and/or increased in severity during the trial, or had an onset date within 30 days after the last dose of study drug.

Neuropathy assessments (PNQ and VPT) were performed at baseline and on day 1 of cycles 2-6, at the beginning of every third cycle thereafter, and at the end of treatment and post-treatment follow-up visits (21 and 42 days after the last dose, respectively). PNQ consisted of three items (sensory, motor, and composite). VPT was measured on the ventral surface of the distal index finger (contralateral to the side of mastectomy or primary disease) and on the distal pad of the right and left great toes, using a Vibratron II device (Physitemp, Inc., Clifton, NJ, USA).

Time to onset of neuropathy was defined as number of weeks from first dose of study treatment to onset of the earliest neuropathy of CTCAE grade greater than baseline for that patient. Time to resolution of neuropathy was defined as number of weeks from last dose of study treatment to the earliest date of resolution of neuropathy (neuropathy that had stopped or resolved to either the level at baseline or lower, and after which there was no further onset).

\section{Efficacy}

Tumor assessments performed at screening/baseline and between days 15 and 21 of cycles 2, 4, and 6 included: computed tomography (CT) scans of the chest, abdomen, and pelvis; a bone scan and/or clinical assessments/photographs of skin lesions; and CT/magnetic resonance imaging scans. In the extension phase, tumor assessments were performed every three cycles until progressive disease (PD) developed.
Tumor response was assessed according to modified Response Evaluation Criteria in Solid Tumors version 1.0, and classified as complete response, partial response (PR), stable disease, or PD. PFS was defined as time from randomization until PD or death due to any cause.

\section{Statistics}

The intent-to-treat population consisted of all randomized patients, and the safety population comprised all patients who received at least one dose of study medication and had at least one post-dose safety assessment. A sample size of 49 patients per group was required to detect a significant difference in the incidence of neuropathy between treatments with $80 \%$ power, at a proportion of 0.35 in the eribulin group to 0.63 in the ixabepilone group (odds ratio 0.316 ), at the 0.05 two-sided significance level.

The primary safety endpoint was analyzed using the Cochran-Mantel-Haenszel test, sensory and motor PNQ scores were analyzed separately using a generalized linear model, and hand and foot VPT data were evaluated by regression analysis. Other neuropathy-related endpoints were not tested for statistical significance. ORR, DCR, and CBR were analyzed using the Clopper-Pearson method, and PFS estimated using Kaplan-Meier analysis.

\section{Results}

Patients

In the intent-to-treat population, 104 patients were randomized equally between the eribulin and ixabepilone groups. The safety analysis set included 101 patients (Fig. 1; Table 1). $32.7 \%$ of patients had received more than three chemotherapy regimens prior to entry. Among those patients with pre-existing neuropathy, the use of medications aimed at symptomatic management of neuropathy during the course of the study was balanced between the two treatment groups ( 4 vs. 3 patients).

Study drug exposure

For the eribulin group, patients received a median number of 5.0 treatment cycles (range 1-30) and spent a median of 15 weeks on treatment (range 3-92) (Table 2). Ixapebilone-treated patients received a median of 3.5 treatment cycles (range 1-15) over a median of 10.5 weeks (range 3-49). The relative dose intensities were similar between the two groups (Table 2). Fewer patients receiving eribulin required dose reductions compared to ixabepilone (21.6 vs. $32.0 \%$ ), although fewer patients receiving ixabepilone experienced dose omissions, delays, or interruptions compared with those receiving eribulin (Table 2). 
Fig. 1 CONSORT diagram

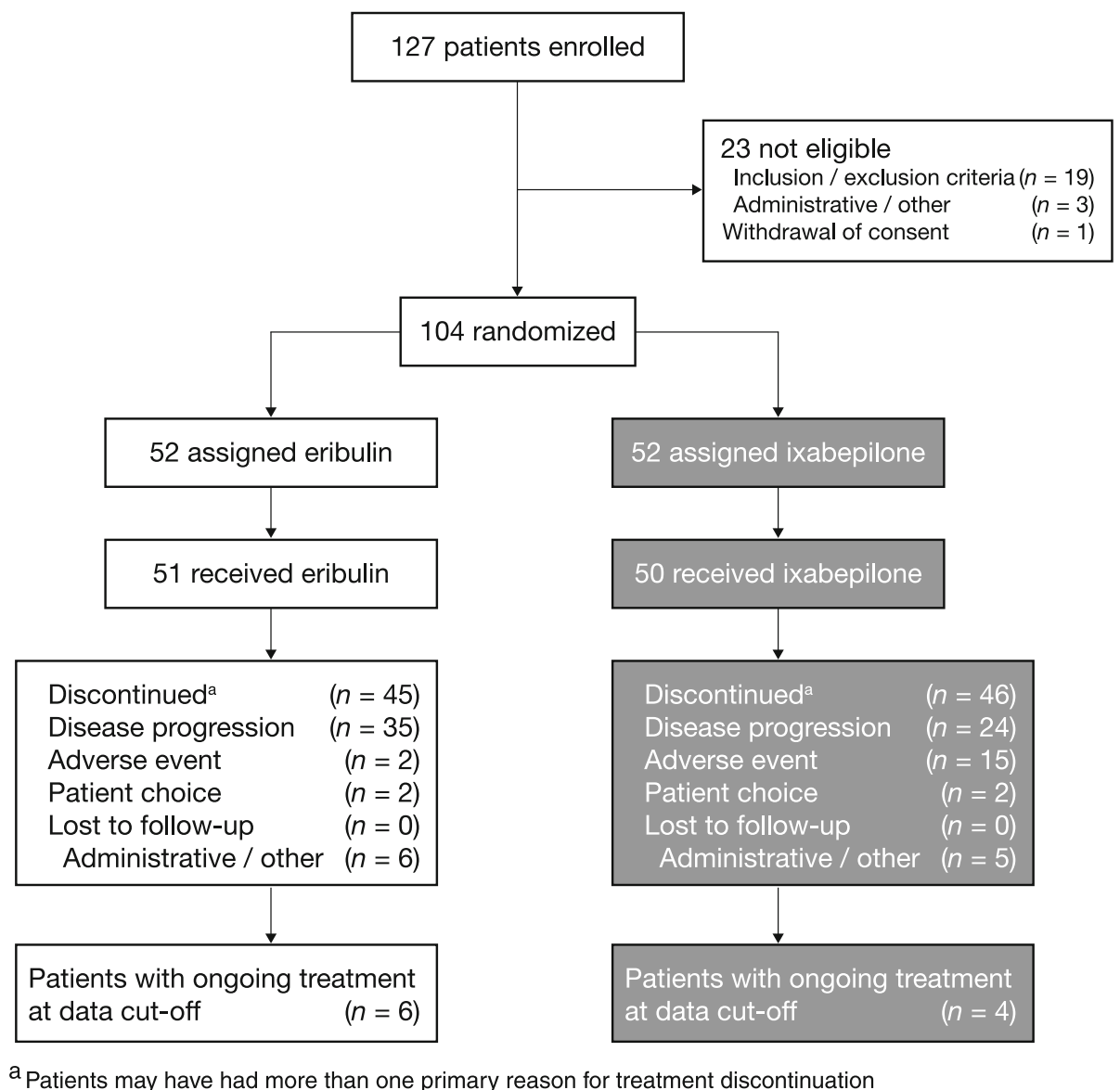

Safety

\section{Neuropathy AEs by CTCAE grade}

Neuropathy of any grade was reported by 17 (33.3\%) and $24(48.0 \%)$ patients receiving eribulin and ixabepilone, respectively (Table 3 ). Sensitivity analysis based on a narrow definition of PN also showed a lower incidence of PN in the eribulin group (16 patients, $31.4 \%$ ) compared with ixabepilone (22 patients, $44.0 \%$ ) (Table 3). After controlling for baseline pre-existing neuropathy (Grade 0 or 1) and number of prior chemotherapies $(\leq 3$, $>3$ ) as binary variables, the difference between treatments in overall incidence of neuropathy and PN was not significant for either $(p=0.1284$ and $p=0.1632$, respectively).

Five $(9.8 \%)$ and $11(22.0 \%)$ eribulin- and ixabepilonetreated patients, respectively, experienced Grade 3 neuropathy. No Grade 4 neuropathy was reported in either group (Table 3). Sensitivity analysis showed a similar incidence of Grade 3 PN (5 [9.8\%] vs. 10 [20.0\%] in patients receiving eribulin and ixabepilone, respectively), with no Grade 4 events reported (Table 3).
Neuropathy AEs by $P N Q$ and vibration sensitivity

No significant differences were observed in change from baseline to worst post-baseline score for motor, sensory, or composite PNQ items between the eribulin and ixabepilone groups.

For vibration sensitivity measurements, the starting threshold values for both the great toe and the index finger were slightly higher (greater deficit) for the ixabepilone group, and the degree of on-study deterioration in sensory function was slightly higher in the eribulin group. However, there were no significant differences in the pattern of VPT findings across treatment groups (Fig. 2).

\section{Time to onset and resolution of neuropathy}

The onset of neuropathy occurred earlier in ixabepilonetreated patients than for those receiving eribulin, with a median time to onset of 11.6 and 35.9 weeks, respectively, from the start of treatment (Fig. 3). By cycle 4, $24.2 \%$ of patients receiving eribulin experienced neuropathy compared with $44.0 \%$ receiving ixabepilone. There were 19 and 12 patients with neuropathy on or after the day of last treatment in the ixabepilone and eribulin groups, respectively, and the 
Table 1 Patient demographics and baseline characteristics (safety population)

\begin{tabular}{|c|c|c|c|}
\hline & Eribulin $(n=51)$ & Ixabepilone $(n=50)$ & Total $(n=101)$ \\
\hline Age (years) [mean (standard deviation)] & $52.2(9.83)$ & $56.9(10.68)$ & $54.5(10.49)$ \\
\hline \multicolumn{4}{|l|}{ Race, $n(\%)$} \\
\hline White & $35(68.6)$ & $44(88.0)$ & $79(78.2)$ \\
\hline Black or African-American & $13(25.5)$ & $5(10.0)$ & $18(17.8)$ \\
\hline Asian & 0 & $1(2.0)$ & $1(1.0)$ \\
\hline Other & $3(5.9)$ & 0 & $3(3.0)$ \\
\hline Time since original diagnosis (years) [mean (standard deviation)] & $6.7(5.4)$ & $7.9(5.6)$ & $7.3(5.5)$ \\
\hline \multicolumn{4}{|l|}{ ECOG performance status, $n(\%)$} \\
\hline 0 & $21(41.2)$ & $20(40.0)$ & $41(40.6)$ \\
\hline 1 & $29(56.9)$ & $28(56.0)$ & $57(56.4)$ \\
\hline 2 & $1(2.0)$ & $2(4.0)$ & $3(3.0)$ \\
\hline \multicolumn{4}{|l|}{ Pre-existing neuropathy, $n(\%)$} \\
\hline CTCAE Grade 0 & $24(47.1)$ & $25(50.0)$ & $49(48.5)$ \\
\hline CTCAE Grade 1 & $27(52.9)$ & $25(50.0)$ & $52(51.5)$ \\
\hline \multicolumn{4}{|l|}{ Hormone receptor status, $n(\%)$} \\
\hline ER positive & $37(72.5)$ & $31(62.0)$ & $68(67.3)$ \\
\hline PgR positive & $27(52.9)$ & $24(48.0)$ & $51(50.5)$ \\
\hline Hormone receptor positive & $37(72.5)$ & $32(64.0)$ & $69(68.3)$ \\
\hline \multicolumn{4}{|l|}{ HER2 status ${ }^{\mathrm{a}}, n(\%)$} \\
\hline HER2 positive & $4(7.8)$ & $7(14.0)$ & $11(10.9)$ \\
\hline Triple negative status (HER2, ER, and PgR negative) & $4(7.8)$ & $11(22.0)$ & $15(14.9)$ \\
\hline \multicolumn{4}{|l|}{ Number of previous chemotherapy regimens in any setting, $n(\%)$} \\
\hline$\leq 3$ & $37(72.5)$ & $31(62.0)$ & $68(67.3)$ \\
\hline$>3$ & $14(27.5)$ & $19(38.0)$ & $33(32.7)$ \\
\hline \multicolumn{4}{|l|}{ Previous chemotherapy, $n(\%)^{\mathrm{b}}$} \\
\hline \multicolumn{4}{|l|}{ Taxanes } \\
\hline Paclitaxel & $38(74.5)$ & $42(84.0)$ & $80(79.2)$ \\
\hline Docetaxel & $25(49.0)$ & $28(56.0)$ & $53(52.5)$ \\
\hline Paclitaxel and docetaxel & $20(40.0)$ & $12(23.5)$ & $32(31.7)$ \\
\hline Doxorubicin & $41(80.4)$ & $37(74.0)$ & $78(77.2)$ \\
\hline Cyclophosphamide & $40(78.4)$ & $36(72.0)$ & $76(75.2)$ \\
\hline Capecitabine & $29(56.9)$ & $33(66.0)$ & $62(61.4)$ \\
\hline Gemcitabine & $17(33.3)$ & $21(42.0)$ & $38(37.6)$ \\
\hline Vinorelbine & $14(27.5)$ & $15(30.0)$ & $29(28.7)$ \\
\hline Carboplatin & $14(27.5)$ & $14(28.0)$ & $28(27.7)$ \\
\hline
\end{tabular}

Safety population: all patients who received at least one dose of study medication and had at least one post-dose safety assessment CTCAE Common Terminology Criteria for Adverse Events, ECOG Eastern Cooperative Oncology Group, ER estrogen receptor, HER2 human epidermal growth factor receptor $2, P g R$ progesterone receptor

a Based on fluorescence in situ hybridization (FISH) or immunochemistry in the absence of FISH testing

b The most frequently reported-incidence $\geq 22 \%$ in one or more treatment groups

maximum time to resolution of neuropathy was shorter for patients treated with ixapebilone ( $n=19 ; 10.1$ weeks; censored) than eribulin ( $n=12 ; 48.4$ weeks; censored).

\section{Adverse events}

The incidences of other AEs were generally comparable between eribulin- and ixabepilone-treated patients
(Table 4). The most common AEs reported were neutropenia $(47.1 \%)$, nausea $(45.1 \%)$, and alopecia $(39.2 \%)$ for eribulin, and fatigue/asthenia $(58.0 \%)$, nausea $(54.0 \%)$, and PN (46.0\%) for ixabepilone (Table 4). A total of 98.0 and $96.0 \%$ of eribulin- and ixabepilone-treated patients, respectively, experienced AEs that were considered related to study drug; of these, 60.8 and $64.0 \%$, respectively, were graded as 3 or higher. Compared with ixabepilone, fewer 
Table 2 Study drug exposure (safety population)

\begin{tabular}{lll}
\hline & Eribulin $(n=51)$ & $\begin{array}{l}\text { Ixabepilone } \\
(n=50)\end{array}$ \\
\hline $\begin{array}{l}\text { Number of cycles received } \\
\text { Mean (standard deviation) }\end{array}$ & $6.2(5.6)$ & $4.8(3.7)$ \\
Median (range) & $5.0(1.0-30.0)$ & $3.5(1.0-15.0)$ \\
Weeks on study medication & & \\
Mean (standard deviation) & $19.8(18.0)$ & $15.0(11.6)$ \\
Median (range) & $15.0(3.0-92.0)$ & $10.5(3.0-49.0)$ \\
Relative dose intensity (\%) & & \\
Mean (standard deviation) & $85.0(16.3)$ & $91.2(11.0)$ \\
Median (range) & $92.3(41.6-103.6)$ & $96.3(49.5-102.5)$ \\
Dose omissions, $n(\%)$ & $9(17.6)$ & $3(6.0)$ \\
Dose reductions, $n(\%)$ & $11(21.6)$ & $16(32.0)$ \\
Dose delays, $n(\%)$ & $29(56.9)$ & $17(34.0)$ \\
Dose interruptions, $n(\%)$ & $6(11.8)$ & $1(2.0)$ \\
\hline
\end{tabular}

Safety population: all patients who received at least one dose of study medication and had at least one post-dose safety assessment

patients receiving eribulin discontinued treatment due to neuropathy (3.9 vs. $18.0 \%$, respectively) or AEs in general (11.8 vs. $32.0 \%)$. Two patients in each group died during the study, all secondary to PD.

\section{Efficacy}

Best overall responses for patients receiving eribulin or ixabepilone are shown in Table 5, and Fig. 4 shows waterfall graphs depicting the change in summed longest diameter of target lesions from baseline to nadir in eribulinand ixabepilone-treated patients. The ORR for eribulin was 15.4 and $5.8 \%$ for ixabepilone (Table 5). Median PFS was 104 days for eribulin-treated patients and 95 days for those receiving ixabepilone.

\section{Discussion}

This is the first clinical study to directly compare neuropathy, safety, and efficacy of eribulin and ixabepilone in patients with MBC. The overall incidences of neuropathy and PN were 33.3 versus $48.0 \%$ and 31.4 versus $44.0 \%$ for eribulin versus ixabepilone, respectively. Regardless of baseline pre-existing neuropathy (Grade 0 or $1)$ and the number of prior chemotherapies $(\leq 3,>3)$, these differences were not significant. The original sample size calculation for the study, to detect a $28 \%$ difference in neuropathy with a power of $80 \%$, was based on historical estimates on the incidence of neuropathy with the two treatments (incidence rates of CTCAE neuropathy events for eribulin [35\%] as per a previous Phase II study [12],
Table 3 Incidence of neuropathy and sensitivity analysis of neuropathy based on a narrow definition of peripheral neuropathy by CTCAE grade (safety population)

\begin{tabular}{lll}
\hline & $\begin{array}{l}\text { Eribulin } \\
(n=51), n(\%)\end{array}$ & $\begin{array}{l}\text { Ixabepilone } \\
(n=50), n(\%)\end{array}$ \\
\hline Neuropathy $^{\mathrm{a}}$ & & $24(48.0)$ \\
All grades & $17(33.3)$ & $13(26.0)$ \\
Grade $1 / 2$ & $12(23.5)$ & $11(22.0)$ \\
Grade 3 & $5(9.8)$ & 0 \\
Grade $\geq 4$ & 0 & $22(44.0)$ \\
Peripheral neuropathy & & $12(24.0)$ \\
All grades & $16(31.4)$ & $10(20.0)$ \\
Grade $1 / 2$ & $11(21.6)$ & 0 \\
Grade 3 & $5(9.8)$ & \\
Grade $\geq 4$ & 0 &
\end{tabular}

Safety population: all patients who received at least one dose of study medication and had at least one post-dose safety assessment

CTCAE Common Terminology Criteria for Adverse Events, MedDRA Medical Dictionary for Regulatory Activities

${ }^{\text {a }}$ Combined MedDRA preferred term based on a broad list of preferred terms defined in standardized MedDRA queries for neuropathy and the following additional preferred terms: neuropathy, hyperesthesia, painful response to normal stimuli, pallanesthesia, and allodynia. If a combined term had $>1$ CTC grade, the highest CTC grade was used

${ }^{\mathrm{b}}$ Of those patients with neuropathy (combined term), peripheral neuropathy was based on a narrow list of preferred terms defined in standardized MedDRA queries for neuropathy and included: neuropathy peripheral, peripheral motor neuropathy, polyneuropathy, peripheral sensory neuropathy, peripheral sensorimotor neuropathy, demyelinating polyneuropathy, and paresthesia

and the package insert for ixabepilone [63\%]). The aforementioned results should, therefore, be interpreted with consideration to the fact that the study was not sufficiently powered to detect the observed magnitude of difference between the two treatment arms.

The severity of neuropathy AEs according to grade supported the overall incidence results, with fewer eribulintreated patients reporting Grade 3/4 neuropathy or PN. There were no significant between-group differences in post-baseline PNQ scores and baseline to worst postbaseline PNQ scores. Additionally, there was no significant effect of time (cycles 2 through 6 or overall) on the correlation between the maximum treatment-emergent motor neuropathy CTC grade and worst post-baseline motor PNQ score. Although the degree of on-study deterioration in sensory function was slightly higher with eribulin, these differences in the pattern of VPT findings across treatment groups were not clinically meaningful or significant. The findings in this study must be tempered by the small number of patients in each group, especially at cycle 6 , and by the substantial variance in the calculated change in VPT scores across patients. 
Fig. 2 Vibration perception threshold scores at baseline, and change from baseline at cycle 3 and 6 in patients receiving eribulin or ixabepilone (safety population)
Fig. 3 Kaplan-Meier analyses of time to onset of neuropathy with eribulin and ixabepilone (safety population)

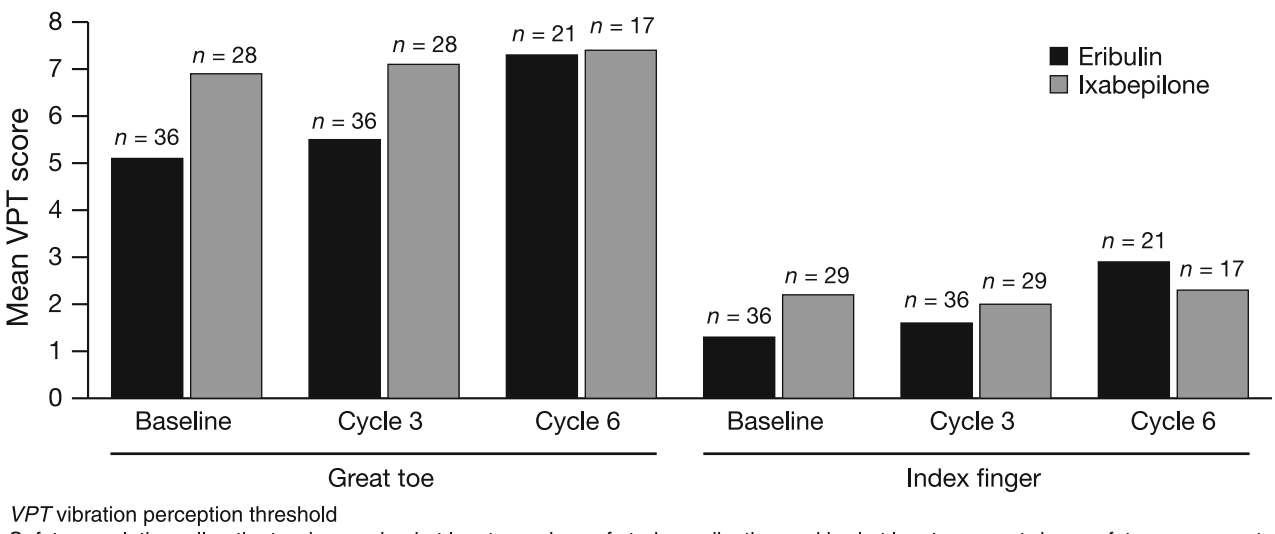

Safety population: all patients who received at least one dose of study medication and had at least one post-dose safety assessment

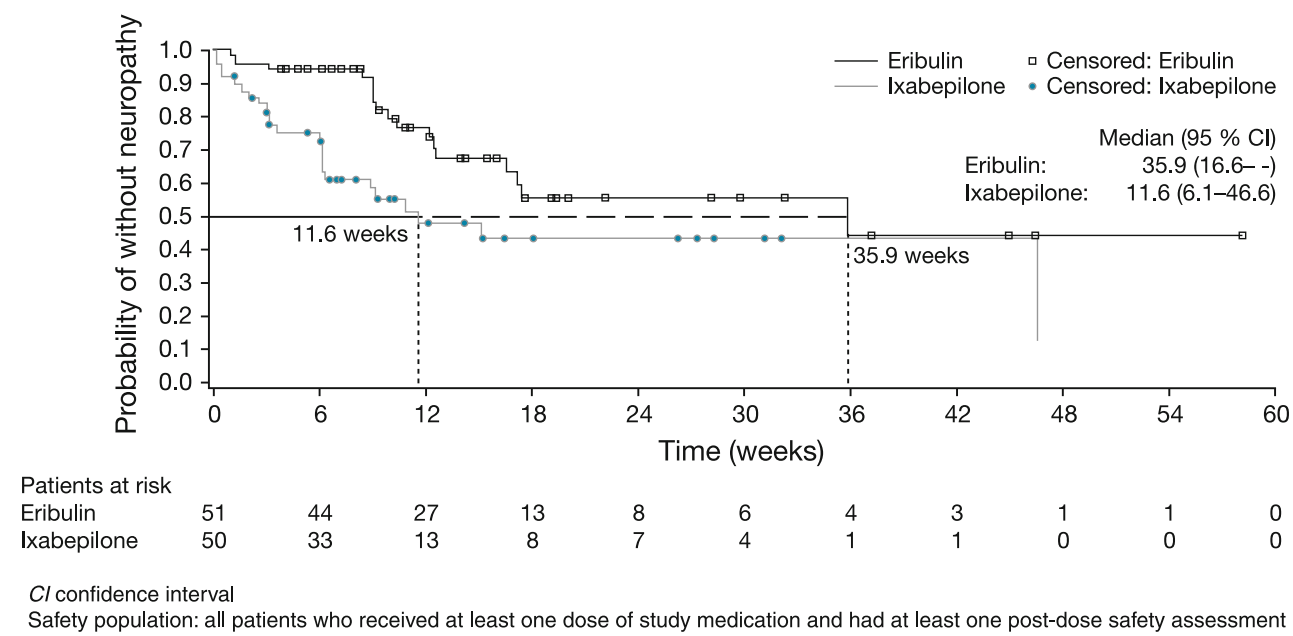

Table 4 Adverse events with an incidence of $>20 \%$ (safety population)

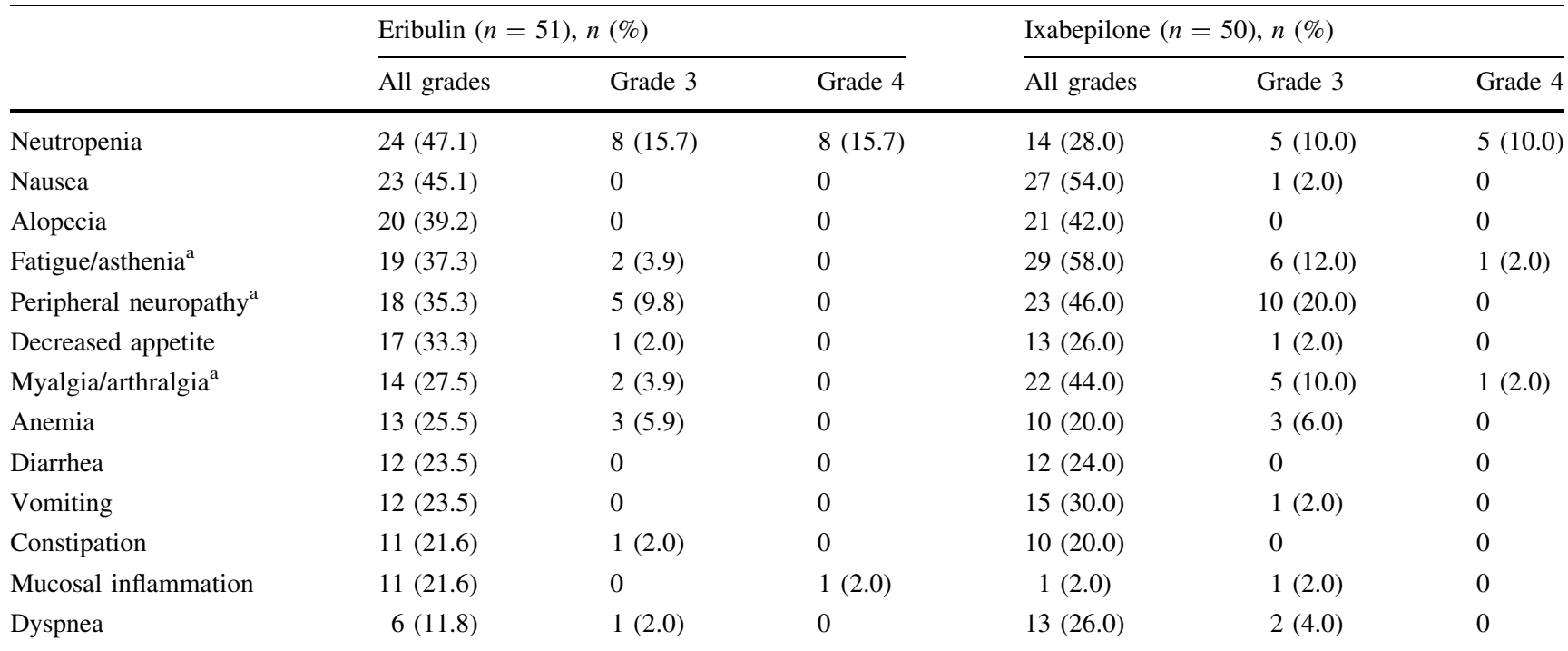

Safety population: all patients who received at least one dose of study medication and had at least one post-dose safety assessment

${ }^{a}$ Combined Medical Dictionary for Regulatory Activities (MedDRA) terms

Eribulin was associated with a longer time to onset of neuropathy in this study. Resolution of neuropathy (in patients with existent neuropathy at the time of treatment discontinuation) tended to occur relatively sooner in patients receiving ixabepilone. However, patients receiving eribulin achieved a greater median number of treatment 
Table 5 Best overall responses and overall response, clinical benefit, and disease control rates with eribulin and ixabepilone (intent-to-treat population)

\begin{tabular}{lll}
\hline & $\begin{array}{l}\text { Eribulin }(n=52), \\
n(\%)\end{array}$ & $\begin{array}{l}\text { Ixabepilone } \\
(n=52), n(\%)\end{array}$ \\
\hline $\begin{array}{l}\text { Best overall response } \\
\text { CR }\end{array}$ & 0 \\
PR & $8(15.4)$ & $3(5.8)$ \\
SD & $27(51.9)$ & $26(50.0)$ \\
PD & $10(19.2)$ & $11(21.2)$ \\
Not evaluable & $7(13.5)$ & $12(23.1)$ \\
ORR (95 \% CI) & $8(15.4)(6.9-28.1)$ & $3(5.8)(1.2-15.9)$ \\
CBR (95 \% CI) & $14(26.9)(15.6-41.0)$ & $10(19.2)(9.6-32.5)$ \\
DCR (95 \% CI) & $35(67.3)(52.9-79.7)$ & $29(55.8)(41.3-69.5)$ \\
Median PFS, days & $104(80.0-129.0)$ & $95(73.0-186.0)$ \\
(95\% CI) & & \\
\hline
\end{tabular}

Intent-to-treat population: all randomized patients

ORR was defined as CR + PR divided by the number of patients in the intent-to-treat population; $\mathrm{CBR}$ was defined as $\mathrm{CR}+\mathrm{PR}+\mathrm{SD}$ $\geq 6$ months; and DCR was $\mathrm{CR}+\mathrm{PR}+\mathrm{SD}$

$C B R$ clinical benefit rate, $C I$ confidence interval, $C R$ complete response, $D C R$ disease control rate, $O R R$ objective response rate, $P D$ progressive disease, $P F S$ progression-free survival, $P R$ partial response, $S D$ stable disease cycles and remained on treatment for longer than those receiving ixabepilone. This raises the possibility that the observed longer time to resolution of neuropathy in patients with ongoing neuropathy at the time of treatment discontinuation in the eribulin group could be attributed to treatment for a longer period than those receiving ixabepilone. A recent pooled analysis of five ixabepilone studies in patients with MBC suggested that PN associated with this agent is cumulative, as the incidence of Grade 3/4 events was shown to increase with median cumulative ixabepilone dose [21]. In addition, PN associated with ixabepilone was shown in monotherapy studies to be generally reversible and often manageable with dose reduction [15-17]. In one such trial, Grade 3/4 PN resolved in $76 \%$ of patients, with a median time to resolution of 5.4 weeks [16]. In EMBRACE, time to onset or resolution of neuropathy were not examined; however, patients with Grade 3/4 PN who continued eribulin treatment also showed improvement following dose modification [7].

In the current study, both agents demonstrated manageable safety profiles. Eribulin exhibited fewer neuropathy-related treatment discontinuations than ixabepilone (3.9 vs. $18.0 \%$ ), which is in line with previously
Fig. 4 Waterfall graphs of change in summed longest diameter of target lesions from baseline to nadir in A eribulinand $\mathbf{B}$ ixabepilone-treated patients

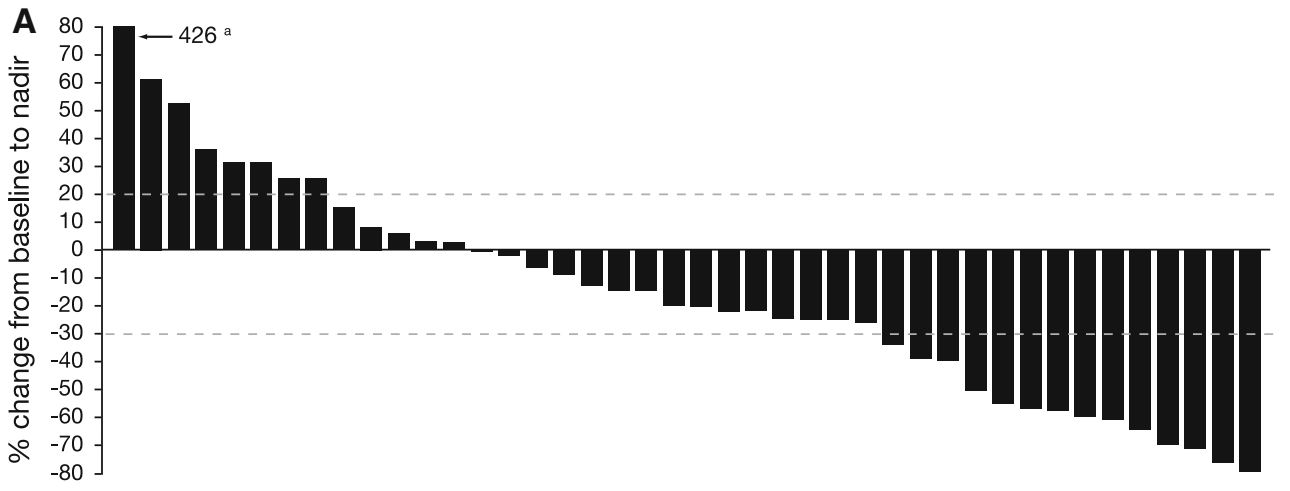

Only patients with target lesion measurement data at both baseline and at least one post-baseline visit are shown

a Patient 10071007 had a baseline value of 23 and only one post-baseline measurement at cycle 2 with a value of 121 , thus the percent change from baseline is $426.1 \%$

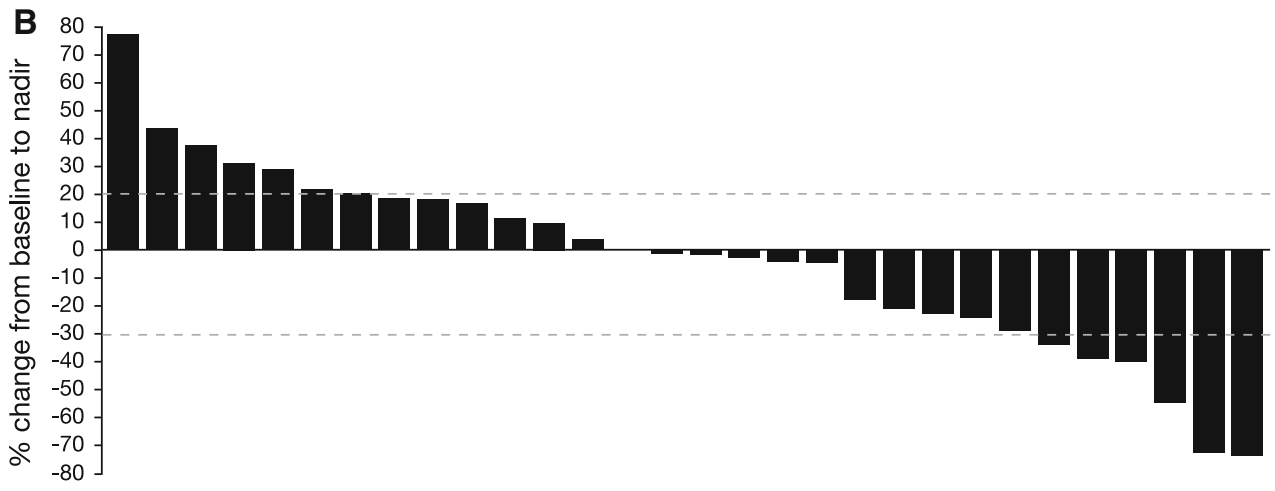

Only patients with target lesion measurement data at both baseline and at least one post-baseline visit are shown 
reported trials. In EMBRACE, treatment discontinuation due to PN occurred in $5 \%$ of patients in the eribulin group [7], whereas monotherapy studies of ixabepilone have reported discontinuations due to neuropathy ranging from 6 to $28 \%$ of patients [15-17]. Discontinuation from treatment due to any toxicity (including neuropathy) was also lower with eribulin than ixabepilone (11.8 vs. $32.0 \%)$.

Due to the small sample size, formal statistical analyses were not planned in the study. In addition, a considerable proportion of patients being either censored or deemed "non evaluable" for efficacy assessments precluded any robust efficacy interpretations from the study. Eribulin, however, had a more favorable impact on ORR than ixabepilone (15.4 vs. $5.8 \%$, respectively) due to a higher proportion of patients exhibiting PRs. In the current study, tumor responses observed with eribulin are similar to those in three previous reports, each of which included patients who had received a median of four previous chemotherapies: in the two earlier Phase II studies of eribulin, ORRs were $9.3 \%$ (95\% CI 6.1-13.4) and $11.5 \%$ (95\% CI 5.7-20.1) [11, 12]; and in EMBRACE, a significantly higher ORR was reported for eribulin $(12 \%$ [95\% CI 9.4-15.5]) compared with TPC (5\% [95\% CI 2.3-8.4]; $p=0.002$ ) [7]. With the median PFS being numerically similar in the eribulin group compared with the ixabepilone group (3.4 vs. 3.1 months, respectively), no clinically meaningful differences in PFS between eribulin and ixabepilone were observed.

In conclusion, differences in the overall incidence of neuropathy were not statistically significant even after controlling for baseline pre-existing neuropathy (Grade 0 or 1$)$ and number of prior chemotherapies $(\leq 3,>3)$. Small sample size and lack of power in the study to detect the observed magnitude of differences may explain why there was no significant numerical differences in the primary safety endpoint between eribulin and ixabepilone. Time to onset of neuropathy tended to occur later and resolve later with eribulin compared with ixabepilone, possibly because patients in the eribulin group received a greater number of treatment cycles and remained on treatment for longer than those in the ixabepilone group. Compared with ixabepilone, fewer patients receiving eribulin discontinued treatment due to neuropathy or AEs in general. Additional studies may be required to confirm whether patients may derive significant benefits with eribulin over ixabepilone with respect to the neuropathy profile.

Acknowledgments We thank all the patients and investigators who participated in these studies. Editorial support was provided by Haley Bennett, $\mathrm{PhD}$, of Complete Medical Communications and was funded by Eisai Inc. This study was funded by Eisai Inc.

Conflict of interest Linda Vahdat has been a consultant/advisor for Eisai, MBS, and Berg. Agustin Garcia has received remuneration from Eisai as a participant in their speakers bureau. Charles Vogel has received remuneration from Aveo, Novartis, Genentech, and Amgen, and his institution (University of Miami) has received funding from Aveo, Genentech, Novartis, and Roche. Christine Pellegrino has received remuneration as a speaker for Eisai. Prashanth Gopalakrishna is affiliated with Eisai Ltd, Hatfield, UK. Joseph Sparano has been a consultant/advisor for Eisai. Deborah Lindquist and Nicholas Iannotti have no conflict of interest to declare.

Ethical standards Approval was obtained from the appropriate regulatory authorities of the United States before sites were initiated. The IEC/IRB and regulatory authorities were notified at the end of the study as defined in the protocol.

Open Access This article is distributed under the terms of the Creative Commons Attribution Noncommercial License which permits any noncommercial use, distribution, and reproduction in any medium, provided the original author(s) and the source are credited.

\section{References}

1. Kuznetsov G, Towle MJ, Cheng H, Kawamura T, TenDyke K, Liu D, Kishi Y, Yu MJ, Littlefield BA (2004) Induction of morphological and biochemical apoptosis following prolonged mitotic blockage by halichondrin B macrocyclic ketone analog E7389. Cancer Res 64:5760-5766

2. Towle MJ, Salvato KA, Budrow J, Wels BF, Kuznetsov G, Aalfs KK, Welsh S, Zheng W, Seletsky BM, Palme MH, Habgood GJ, Singer LA, Dipietro LV, Wang Y, Chen JJ, Quincy DA, Davis A, Yoshimatsu K, Kishi Y, Yu MJ, Littlefield BA (2001) In vitro and in vivo anticancer activities of synthetic macrocyclic ketone analogues of halichondrin B. Cancer Res 61:1013-1021

3. Jordan MA, Kamath K, Manna T, Okouneva T, Miller HP, Davis C, Littlefield BA, Wilson L (2005) The primary antimitotic mechanism of action of the synthetic halichondrin E7389 is suppression of microtubule growth. Mol Cancer Ther 4:1086-1095

4. Okouneva T, Azarenko O, Wilson L, Littlefield BA, Jordan MA (2008) Inhibition of centromere dynamics by eribulin (E7389) during mitotic metaphase. Mol Cancer Ther 7:2003-2011

5. Smith JA, Wilson L, Azarenko O, Zhu X, Lewis BM, Littlefield BA, Jordan MA (2010) Eribulin binds at microtubule ends to a single site on tubulin to suppress dynamic instability. Biochemistry 49:1331-1337

6. Towle MJ, Salvato KA, Wels BF, Aalfs KK, Zheng W, Seletsky BM, Zhu X, Lewis BM, Kishi Y, Yu MJ, Littlefield BA (2011) Eribulin induces irreversible mitotic blockade: implications of cell-based pharmacodynamics for in vivo efficacy under intermittent dosing conditions. Cancer Res 71:496-505

7. Cortes J, O'Shaughnessy J, Loesch D, Blum JL, Vahdat LT, Petrakova K, Chollet P, Manikas A, Diéras V, Delozier T, Vladimirov V, Cardoso F, Koh H, Bougnoux P, Dutcus CE, Seegobin S, Mir D, Meneses N, Wanders J, Twelves C (2011) Eribulin monotherapy versus treatment of physician's choice in patients with metastatic breast cancer (EMBRACE): a Phase 3 open-label randomised study. Lancet 377:914-923

8. Eisai Inc (2012) Halaven prescribing information. http://www. halaven.com/. Accessed 1 Feb 2013

9. Pachman DR, Barton DL, Watson JC, Loprinzi CL (2011) Chemotherapy-induced peripheral neuropathy: prevention and treatment. Clin Pharmacol Ther 90:377-387

10. Speck RM, Demichele A, Farrar JT, Hennessy S, Mao JJ, Stineman MG, Barg FK (2012) Scope of symptoms and self-management strategies for chemotherapy-induced peripheral 
neuropathy in breast cancer patients. Support Care Cancer 20:2433-2439

11. Vahdat LT, Pruitt B, Fabian CJ, Rivera RR, Smith DA, Tan-Chiu E, Wright J, Tan AR, Dacosta NA, Chuang E, Smith J, O'Shaughnessy J, Shuster DE, Meneses NL, Chandrawansa K, Fang F, Cole PE, Ashworth S, Blum JL (2009) Phase II study of eribulin mesylate, a halichondrin B analog, in patients with metastatic breast cancer previously treated with an anthracycline and a taxane. J Clin Oncol 27:2954-2961

12. Cortes J, Vahdat L, Blum JL, Twelves C, Campone M, Roché H, Bachelot T, Awada A, Paridaens R, Goncalves A, Shuster DE, Wanders J, Fang F, Gurnani R, Richmond E, Cole PE, Ashworth S, Allison MA (2010) Phase II study of the halichondrin B analog eribulin mesylate in patients with locally advanced or metastatic breast cancer previously treated with an anthracycline, a taxane, and capecitabine. J Clin Oncol 28:3922-3928

13. Lee FY, Borzilleri R, Fairchild CR, Kim SH, Long BH, Reventos-Suarez C, Vite GD, Rose WC, Kramer RA (2001) BMS247550: a novel epothilone analog with a mode of action similar to paclitaxel but possessing superior antitumor efficacy. Clin Cancer Res 7:1429-1437

14. Bristol-Myers Squibb Company (2011) IXEMPRA ${ }^{\mathrm{TM}}$ Kit (ixabepilone) for Injection prescribing information. http://packageinserts. bms.com/pi/pi_ixempra.pdf. Accessed 1 Feb 2013

15. Thomas E, Tabernero J, Fornier M, Conté P, Fumoleau P, Lluch A, Vahdat LT, Bunnell CA, Burris HA, Viens P, Baselga J, Rivera E, Guarneri V, Poulart V, Klimovsky J, Lebwohl D, Martin M (2007) Phase II clinical trial of ixabepilone (BMS-247550), an epothilone B analog, in patients with taxane-resistant metastatic breast cancer. J Clin Oncol 25:3399-3406

16. Perez EA, Lerzo G, Pivot X, Thomas E, Vahdat L, Bosserman L, Viens P, Cai C, Mullaney B, Peck R, Hortobagyi GN (2007) Efficacy and safety of ixabepilone (BMS-247550) in a Phase II study of patients with advanced breast cancer resistant to an anthracycline, a taxane, and capecitabine. J Clin Oncol 25:3407-3414

17. Roché H, Yelle L, Cognetti F, Mauriac L, Bunnell C, Sparano J, Kerbrat P, Delord JP, Vahdat L, Peck R, Lebwohl D, Ezzeddine R, Curé H (2007) Phase II clinical trial of ixabepilone (BMS247550), an epothilone B analog, as first-line therapy in patients with metastatic breast cancer previously treated with anthracycline chemotherapy. J Clin Oncol 25:3415-3420

18. Rugo HS, Barry W, Moreno-Aspitia A, Lyss AP, Cirrincione C, Mayer EL, Naughton M, Layman RM, Carey LA, Somer RA, Perez EA, Hudis C, Winer EP (2012) CALGB 40502/NCCTG N063H: Randomized Phase III trial of weekly paclitaxel (P) compared to weekly nanoparticle albumin bound nab-paclitaxel (NP) or ixabepilone (Ix) with or without bevacizumab (B) as first-line therapy for locally recurrent or metastatic breast cancer (MBC). J Clin Oncol 30:CRA1002

19. Wozniak KM, Nomoto K, Lapidus RG, Wu Y, Carozzi V, Cavaletti G, Hayakawa K, Hosokawa S, Towle MJ, Littlefield BA, Slusher BS (2011) Comparison of neuropathy-inducing effects of eribulin mesylate, paclitaxel, and ixabepilone in mice. Cancer Res 71:3952-3962

20. LaPointe NE, Wilson L, Feinstein SC, Jordan M (2011) Effects of eribulin, vincristine, paclitaxel, and ixabepilone on kinesin-driven microtubule gliding. Presented at Society for Neuroscience National Meeting, Washington DC, USA, 12-16 Nov 2011, poster 367.01

21. Vahdat LT, Thomas ES, Roché HH, Hortobagyi GN, Sparano JA, Yelle L, Fornier MN, Martin M, Bunnell CA, Mukhopadhyay P, Peck RA, Perez EA (2012) Ixabepilone-associated peripheral neuropathy: data from across the Phase II and III clinical trials. Support Care Cancer 20:2661-2668 